\title{
Calculation of Parameter of the Ashcroft Model Potential for Hexagonal Closed Pack (hcp) Crystals
}

\author{
A. GhORAI* \\ Department of Physics; Maulana Azad College 8, Rafi Ahmed Kidwai Road, Kolkata — 700013, India \\ (Received January 29, 2018; revised version July 4, 2018; in final form July 12, 2018) \\ Parameter of the Ashcroft model potential has been computed in this paper for twenty two hexagonal closed \\ pack (hcp) crystals. Calculation uses pseudopotential technique with nine different exchange and correlation \\ functions and either available experimental value of monovacancy formation energy or an empirical relation based on \\ other experimental parameters (melting temperature, cohesive energy or activation energy) as tool. The complete \\ set of value of this parameter for cubic crystals will be used for further calculation of energetic of self and impurity \\ diffusion via vacancy mechanism or other type of point defects.
}

DOI: 10.12693/APhysPolA.134.549

PACS/topics: point defect, vacancy, pseudopotential, Ashcroft model, hcp crystal

\section{Introduction}

The pseudopotential approach has been tested earlier several times [1-3] and in this paper this formalism is applied to twenty two different hexagonal closed pack (hcp) crystals, viz. (1) beryllium (Be), (2) magnesium (Mg), (3) scandium (Sc), (4) titanium (Ti), (5) cobalt (Co), (6) zinc $(\mathrm{Zn}),(7)$ yttrium $(\mathrm{Y})$, (8) zirconium (Zr), (9) technetium (Tc), (10) ruthenium (Ru), (11) cadmium (Cd), (12) gadolinium $(\mathrm{Gd})$, (13) terbium $(\mathrm{Tb})$, (14) dysprosium (Dy),(15) holmium (Ho), (16) erbium (Er), (17) thullium (Tm), (18) lutetium (Lu), (19) hafnium (Hf), (20) rhenium (Re), (21) osmium (Os) and (22) thallium (Tl) (arranged according to increasing atomic number). Although ab initio, DFT, molecular dynamics, etc. calculations are most sophisticated techniques yet they lack exact predictions when applied to defect property calculations. In this situation this work is important because rigorous computation is not required as in above new methodologies. Secondly it is comparatively easier technique and thirdly there are very few data of point defect parameters for hcp crystals. Especially out of 22 hcp crystals experimental evidence of monovacancy formation energy is obtained for only six. So an empirical relation among cohesive energy, melting temperature, activation energy for self diffusion, and monovacancy formation energy is used for theoretical estimation. Also it is important because of recent studies of the mechanism of melting $[4,5]$ by considering the role of surfaces with regard to the concentration and migration of vacancies. Lattice instability occurs both at the surface and within the crystal lattice when the vacancy concentration increases from $0.37 \%$ to $10 \%$ on melting.

\section{Formulations}

Harrison's second order perturbation theory gives the total energy of the pure crystal as [3]:

*e-mail: amitavaghorai@rediffmail.com

$$
E_{T}=z\left[\frac{3 \hbar^{2} k_{\mathrm{F}}^{2}}{10 m}+\langle\boldsymbol{k}|W(r)| \boldsymbol{k}\rangle\right]+E_{b s}+E_{e s} .
$$

Here $z$ is the valency, $k_{\mathrm{F}}$ - the Fermi wave number, $m$ - the electronic mass, and $W(r)$ - the pseudopotential. The first term within square bracket is structure independent and the last two terms, called the electrostatic energy $E_{e s}$ (depends on ion-ion interaction) and the band structure energy $E_{b s}$ (depends on ionelectron and electron-electron interactions) depend on the crystal structure. Any defect in the lattice changes the structure dependent energy part of $E_{T}$ and so an algebraic difference between the energy after defect creation and that before will yield the defect formation energy when considered for the whole lattice. This structure dependent part of $E_{T}$ also depends on the modified lattice wave numbers. The modifications in the lattice wave numbers from its perfect lattice value, is necessary to maintain the lattice volume and the number of lattice ions constant. Details of it can be obtained from literature and finally the expression for vacancy formation energy is

$$
E_{\mathrm{F}}^{1 v}=\sum_{q_{0}}^{\prime} \frac{q_{0}}{3} \frac{\partial U\left(q_{0}\right)}{\partial q_{0}}+\frac{\Omega}{2 \pi^{2}} \int_{0}^{\propto} U(q) q^{2} \mathrm{~d} q,
$$

where

$$
U(q)=\lim _{\eta \rightarrow \infty} \frac{2 \pi z^{2} e^{2}}{\Omega q^{2}} \mathrm{e}^{-\frac{q^{2}}{4 \eta}}+[\omega(q)]^{2} \varepsilon(q) \chi(q)
$$

Here $\eta$ is the convergence factor, $\Omega$ - the atomic volume, $\boldsymbol{q}_{0}$ and $\boldsymbol{q}$ - the lattice and quasi-continuous wave numbers respectively, $\omega(q)$ - the pseudopotential, $\varepsilon(q)$ - the dielectric function, and $\chi(q)$ - the perturbation characteristics. Here I have considered the Ashcroft model pseudopotential [6], which is

$$
w(q)=-\frac{4 \pi z e^{2} \cos q r_{c}}{\Omega q^{2}} .
$$

Here $r_{c}$ is the parameter for the Ashcroft model. The expressions for $\varepsilon(q)$ and $\chi(q)$ are

$$
\varepsilon(q)=1-\frac{8 \pi \mathrm{e}^{2}}{\Omega q^{2}}[1-f(q)] \chi(q)
$$




$$
\chi(q)=-\frac{m k_{\mathrm{F}} \Omega}{4 \pi^{2} \hbar^{2}}\left(1+\frac{4 k_{\mathrm{F}}^{2}-q^{2}}{4 k_{\mathrm{F}} q} \ln \frac{\left|2 k_{\mathrm{F}}+q\right|}{\left|2 k_{\mathrm{F}}-q\right|}\right)
$$

Here $f(q)$ is the exchange and correlation function (hence forth called ECF). Out of several ECFs only nine such forms are taken into account and the expressions [1] are

King and Kutler ECF (abbreviated henceforth as K-K)

$$
f(q)=\frac{q^{2}}{2\left(2 k_{\mathrm{F}}^{2}+q^{2}\right)},
$$

Sham ECF (abbreviated as Sham)

$$
f(q)=\frac{q^{2}}{2\left(k_{\mathrm{F}}^{2}+q^{2}+\frac{2 k_{\mathrm{F}}}{\pi a_{0}}\right)},
$$

Geldart and Vosko ECF (abbreviated as G-V)

$$
f(q)=\frac{q^{2}}{2 q^{2}+4 k_{\mathrm{F}}^{2} /\left(1+\frac{0.026 m^{\prime}}{m} \sqrt[3]{\frac{3 \Omega}{4 \pi z a_{0}^{3}}}\right)},
$$

Kleinmann ECF (abbreviated as Kle)

$$
f(q)=\frac{1}{4}\left(\frac{q^{2}}{k_{\mathrm{F}}^{2}+\frac{2 k_{\mathrm{F}}}{\pi a_{0}}}+\frac{q^{2}}{k_{\mathrm{F}}^{2}+q^{2}+\frac{2 k_{\mathrm{F}}}{\pi a_{0}}}\right),
$$

Harrison ECF (abbreviated as Harr)

$$
f(q)=\frac{q^{2}}{2\left(q^{2}+\frac{4 k_{\mathrm{F}}^{2}}{3}\right)},
$$

Vashishta and Singwi ECF (abbreviated as V-S)

$$
f(q)=A\left(1-\mathrm{e}^{-\frac{B q^{2}}{k_{\mathrm{F}}^{2}}}\right),
$$

Taylor ECF (abbreviated as Tay)

$$
f(q)=\frac{q^{2}}{4 k_{\mathrm{F}}^{2}}\left(1+\frac{0.1534}{\pi k_{\mathrm{F}}}\right),
$$

Hubbard ECF (abbreviated as Hub)

$$
f(q)=\frac{q^{2}}{2\left(k_{\mathrm{F}}^{2}+q^{2}\right)},
$$

Mahanti and Das ECF (abbreviated as M-D)

$$
\begin{gathered}
f(q)=\frac{q^{2}}{k_{\mathrm{F}}^{2}+4 k_{\mathrm{F}}^{2} /\left(1+\frac{0.026 m^{\prime}}{m} \sqrt[3]{\frac{3 \Omega}{4 \pi z a_{0}^{3}}}\right)} \\
+\frac{q^{2}}{k_{\mathrm{F}}^{2}+q^{2}+4 k_{\mathrm{F}}^{2} /\left(1+\frac{0.026 m^{\prime}}{m} \sqrt[3]{\frac{3 \Omega}{4 \pi z a_{0}^{3}}}\right)} .
\end{gathered}
$$

Here $a_{0}$ is the Bohr radius. Computation has been done by integration over quasi-continuous wave numbers $\boldsymbol{q}$ using the Gauss-Legendre quadrature integration within the limit from 0 to 1 in 100 divisions and Gauss-Laguerre quadrature integration in the limit from 1 to infinity as follows:

$$
\int_{0}^{\propto} \rightarrow \int_{0}^{1} \text { Gauss-Legendre }+\int_{1}^{\propto} \text { Gauss-Laguerre. }
$$

Computation also uses a discrete sum over lattice wave numbers $\boldsymbol{q}_{0}$ with primitive and reciprocal lattice vectors are defined respectively as

$$
\begin{aligned}
& \boldsymbol{a}_{1}=\frac{\sqrt{3} a}{2} \widehat{i}+\frac{a}{2} \widehat{j}, \quad \boldsymbol{a}_{2}=-\frac{\sqrt{3} a}{2} \widehat{i}+\frac{a}{2} \widehat{j}, \\
& \boldsymbol{a}_{3}=c \widehat{k}
\end{aligned}
$$

$$
\begin{aligned}
\boldsymbol{q}_{1} & =\frac{2 \pi}{\sqrt{3} a}(\widehat{i}+\sqrt{3} \widehat{j}), \quad \boldsymbol{q}_{2}=\frac{2 \pi}{\sqrt{3} a}(-\widehat{i}+\sqrt{3} \widehat{j}), \\
\boldsymbol{q}_{3} & =\frac{2 \pi}{c} \widehat{k}
\end{aligned}
$$

with

$$
\boldsymbol{q}_{0}=\frac{m_{1}}{N_{1}} \boldsymbol{q}_{1}+\frac{m_{2}}{N_{2}} \boldsymbol{q}_{2}+\frac{m_{3}}{N_{3}} \boldsymbol{q}_{3} .
$$

The maximum value of $m_{i} / N_{i}=14$ with $i=1,2,3$ and the lattice wave numbers are generated in the cubic Brillouin zone. Here $a$ and $c$ are lattice constants of hcp crystal.

\section{Discussions}

The input parameters used in this calculation are lattice constant ( $a$ and $c$ ) and experimental value of vacancy formation energy $\left(E_{\mathrm{F}}^{1 v}\right)_{\exp }$ or theoretical mean value of it $\left(E_{\mathrm{F}}^{1 v}\right)_{\text {fit }}$. The necessity of $\left(E_{\mathrm{F}}^{1 v}\right)_{\text {fit }}$ is due to the fact that in most of the cases the experimental value of $E_{\mathrm{F}}^{1 v}$ is not available and so $\left(E_{\mathrm{F}}^{1 v}\right)_{f i t}$ is determined from an empirical relation among the melting temperature $\left(T_{m}\right)$, the cohesive energy $\left(E_{\mathrm{coh}}\right)$, the activation energy $\left(Q_{0}\right)$ and monovacancy formation energy $\left(E_{\mathrm{F}}^{1 v}\right)$ as

$$
\begin{aligned}
& T_{m}(\mathrm{~K})=1200 E_{\mathrm{F}}^{1 v}(\mathrm{eV})=660 Q_{0}(\mathrm{eV})= \\
& 360 E_{\mathrm{coh}}(\mathrm{eV}) .
\end{aligned}
$$

Earlier several researchers used different experimental results, viz. resistivity, phonon dispersion relation, etc. to determine parameter of the Ashcroft model potential $r_{c}$ and here experimental or mean theoretical estimation of $E_{\mathrm{F}}^{1 v}$ is used to determine $r_{c}$. Comparison is done with available other theoretical values of $E_{\mathrm{F}}^{1 v}$ which are shown in Table I. Our fitted value of $\left(E_{\mathrm{F}}^{1 v}\right)_{\text {fit }}$ is very close to the Angsten et al. estimated values [8].

The variation of $E_{\mathrm{F}}^{1 v}$ in rydbergs $(1 \mathrm{Ry}=13.605 \mathrm{eV})$ with the Ashcroft parameter $r_{c}$ are shown in Figs. 14 within zero to $5 \mathrm{AU}(1 \mathrm{AU}=0.0529177 \mathrm{~nm})$. The graphs are almost similar for all exchange and correlation functions because of square of cosine term of the Ashcroft model. The available experimental value of $E_{\mathrm{F}}^{1 v}$ is found to lie near the first nodal point corresponding to the condition $E_{\mathrm{F}}^{1 v} \rightarrow 0$ and $a_{0} \leq r_{c}<2 A U$ where $a_{0}(=1 \mathrm{AU})$ is the Bohr radius. The fitted value of $r_{c}$ is shown in Table II for different ECFs. 

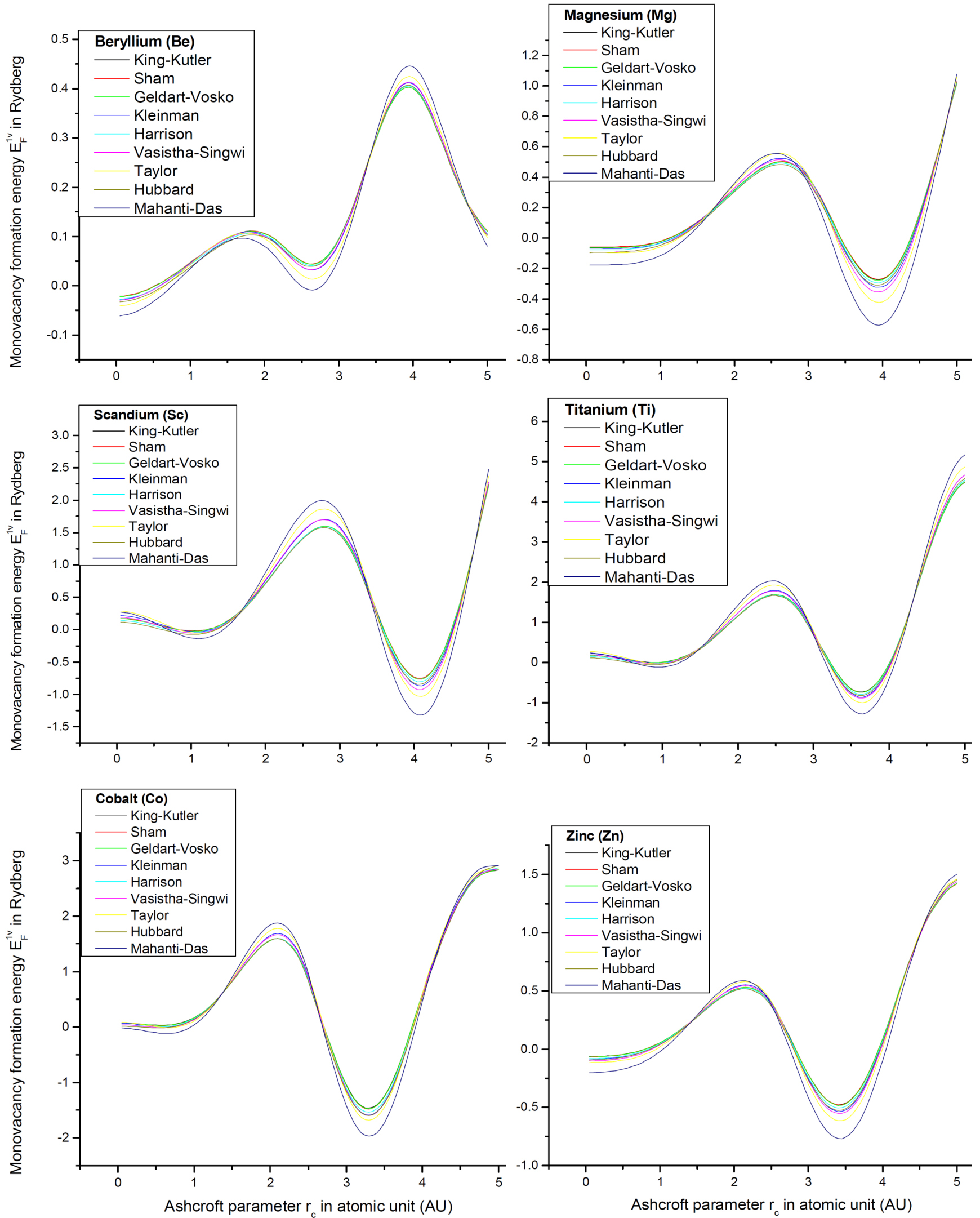

Fig. 1. $\quad E_{\mathrm{F}}^{1 v}-r_{c}$ plots for for beryllium (Be), magnesium (Mg), scandium (Sc), titanium (Ti), cobalt (Co), zinc (Zn). 

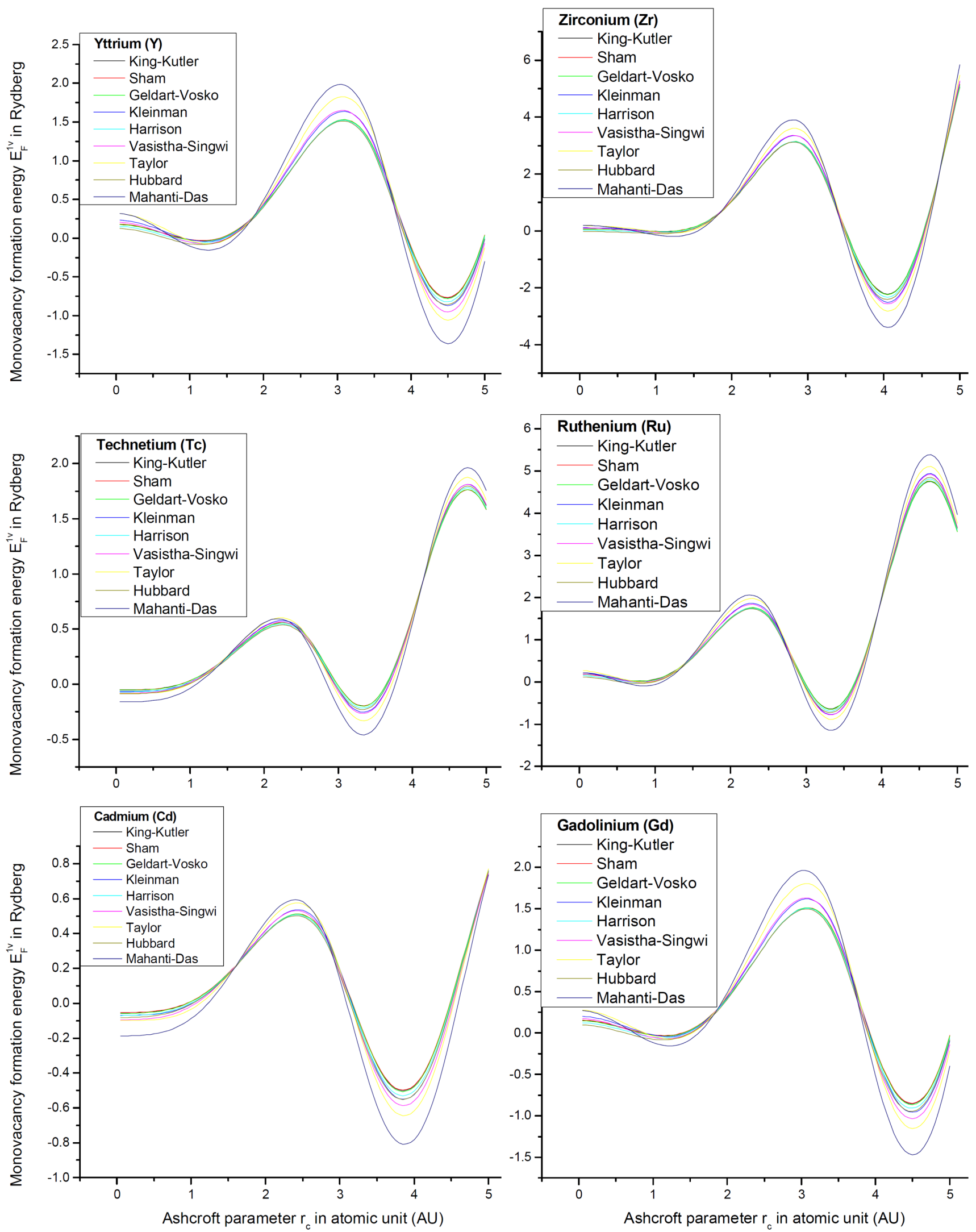

Fig. 2. $\quad E_{\mathrm{F}}^{1 v}-r_{c}$ plots for yttrium (Y), zirconium $(\mathrm{Zr})$, technetium (Tc), ruthenium (Ru), cadmium (Cd), gadolinium (Gd). 

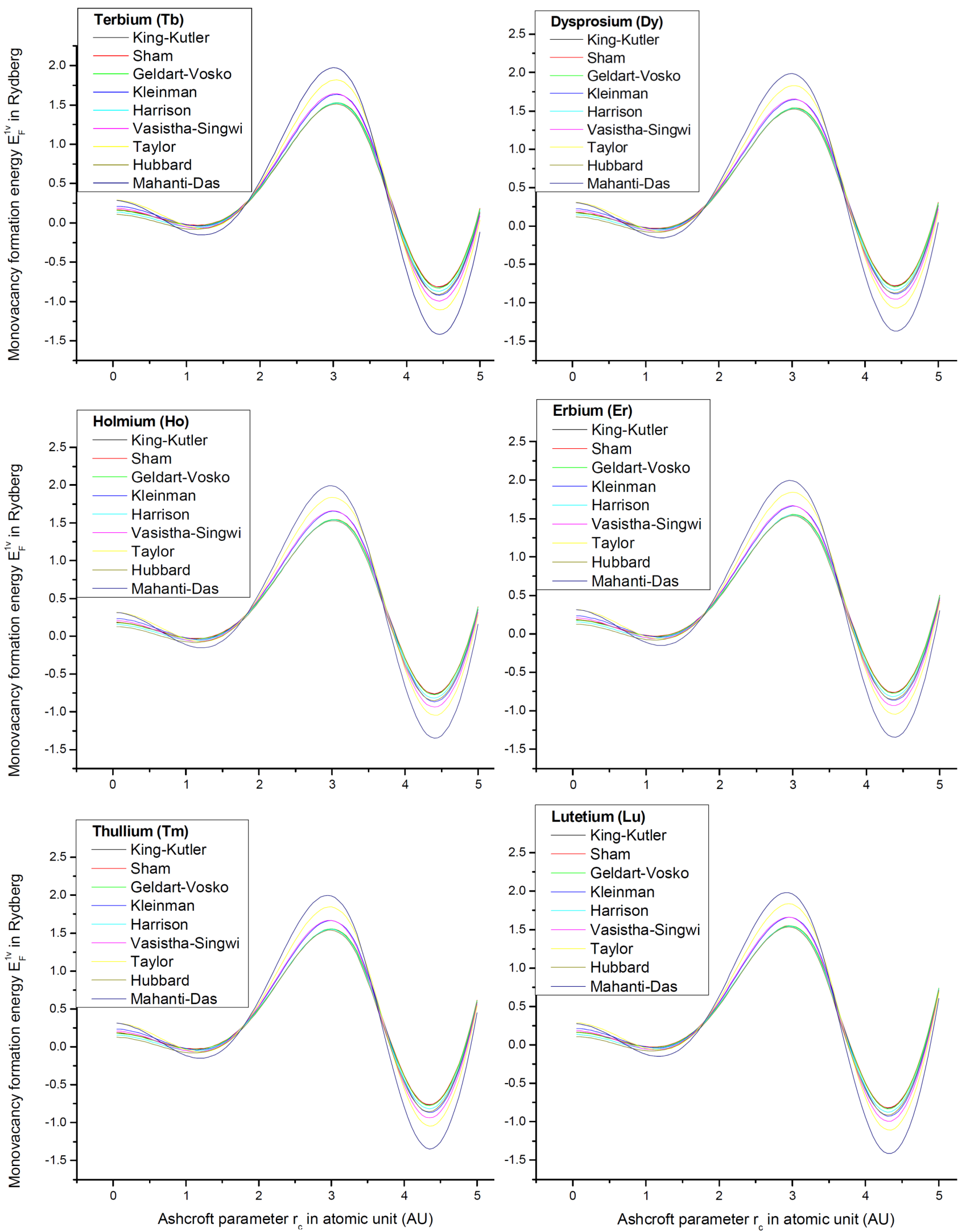

Fig. 3. $\quad E_{\mathrm{F}}^{1 v}-r_{c}$ plots for terbium (Tb), dysprosium (Dy), holmium (Ho), erbium (Er), thulium (Tm), lutetium (Lu). 

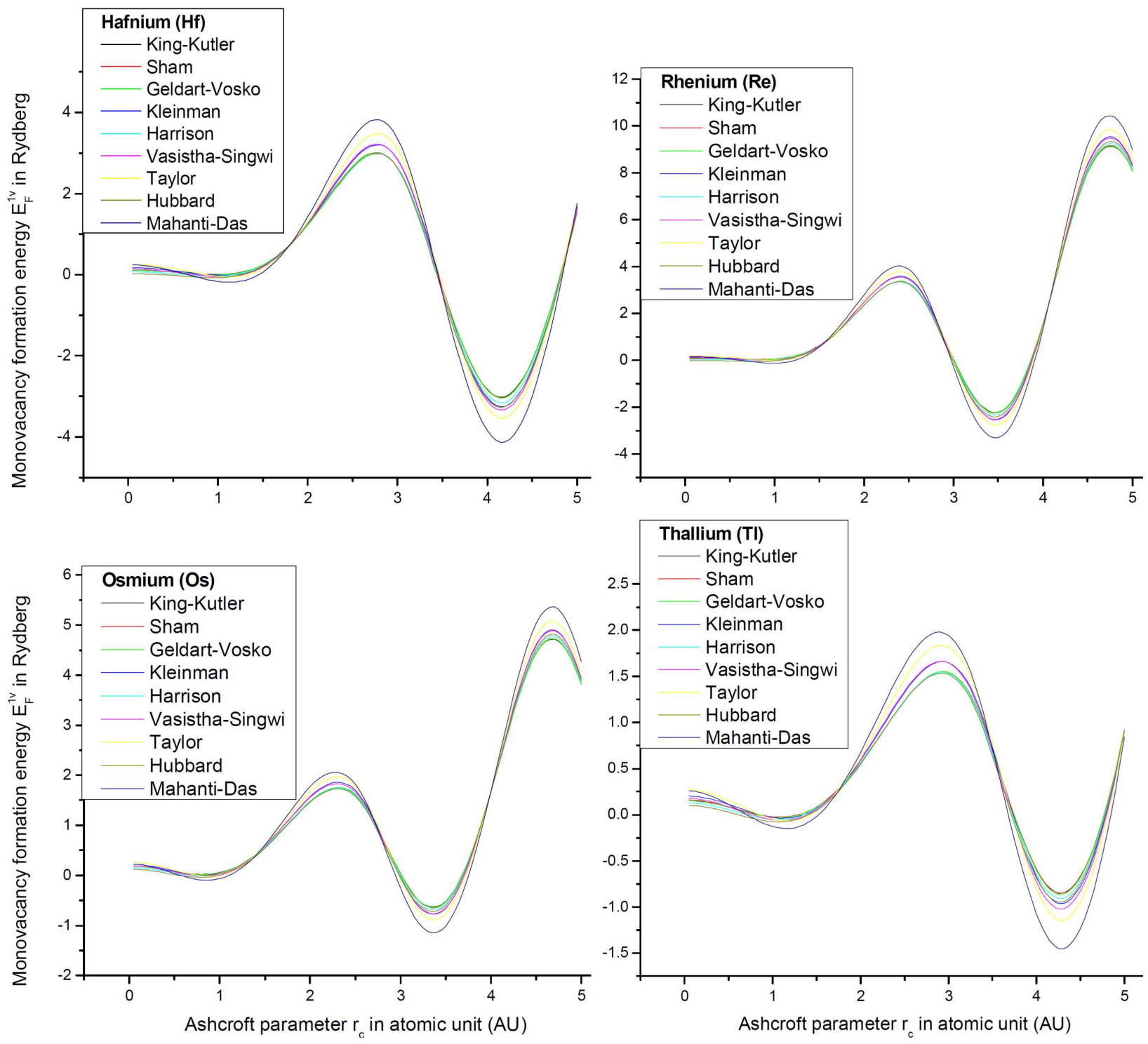

Fig. 4. $E_{\mathrm{F}}^{1 v}-r_{c}$ plots for hafnium (Hf), rhenium (Re) IV, osmium (Os) III, thallium (Tl) III.

The correct value of valency in crystalline state has been chosen out of multiple values by noting that the Ashcroft parameter $r_{c}$ lies near to first peak and the Bohr radius. A graph shown in Fig. 5 for atomic number and fitted $r_{c}$ for different fcc [1] and hcp cubic crystals satisfies this idea. Thus out of two valence states $(1,2)$ for beryllium $z=1$ has been chosen. Similarly for other multi-valent metals like titanium, cobalt, technetium, ruthenium, terbium, rhenium, osmium and thallium the valency values corresponding to lowest $r_{c}$ values are listed in Table I.

The variation of the Ashcroft parameter $r_{c}$ with different exchange and correlation functions for different hcp crystals is shown in Table II with the mean value. The value of $r_{c}$ in cases of cobalt and zinc show slightly less than the Bohr radius. This may be due to less estimation of $\left(E_{\mathrm{F}}^{1 v}\right)_{\text {fit }}$ because of higher calculated values

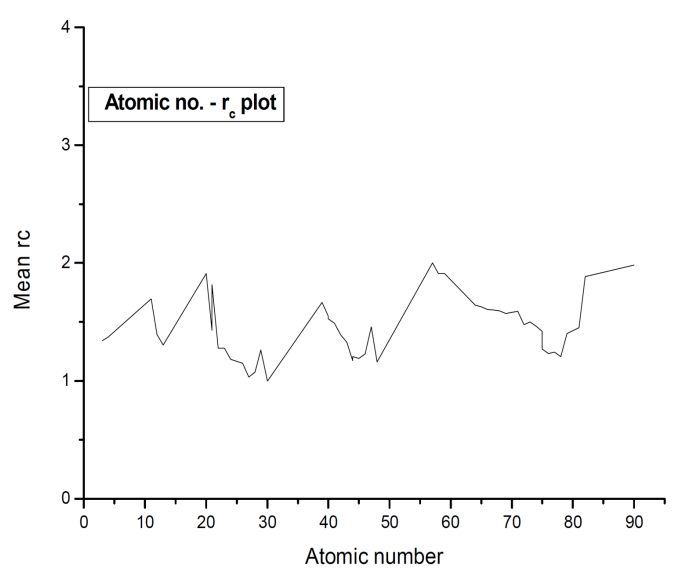

Fig. 5. Mean Ashcroft parameter $r_{c}$ for hcp and fcc crystals. 
Input parameters for hexagonal closed pack (hcp) crystals

TABLE I

\begin{tabular}{|c|c|c|c|c|c|c|c|c|c|c|c|c|}
\hline \multirow{2}{*}{ Element } & \multirow{2}{*}{ Atomic no. } & \multirow{2}{*}{$z$} & \multirow{2}{*}{$a^{a}[\mathrm{~nm}]$} & \multirow{2}{*}{$c^{a}[\mathrm{~nm}]$} & \multicolumn{2}{|c|}{ V-S parameters } & \multirow{2}{*}{$\begin{array}{l}T_{m}^{a} \\
{[\mathrm{~K}]}\end{array}$} & \multirow{2}{*}{\begin{tabular}{l|}
$E_{\mathrm{coh}}^{a}$ \\
{$[\mathrm{eV}]$} \\
\end{tabular}} & \multirow{2}{*}{$\begin{array}{c}Q_{0}^{b} \\
{[\mathrm{eV}]}\end{array}$} & \multirow{2}{*}{$\begin{array}{c}\left(E_{\mathrm{F}}^{1 v}\right)_{\mathrm{fit}} \\
{[\mathrm{eV}]}\end{array}$} & \multirow{2}{*}{$\begin{array}{c}\left(E_{\mathrm{F}}^{1 v}\right)_{\mathrm{Th}}^{b} \\
{[\mathrm{eV}]}\end{array}$} & \multirow{2}{*}{$\begin{array}{c}\left(E_{\mathrm{F}}^{1 v}\right)_{\exp } \\
{[\mathrm{eV}]}\end{array}$} \\
\hline & & & & & $\mathrm{A}$ in $\mathrm{AU}$ & $\mathrm{B}$ in $\mathrm{AU}$ & & & & & & \\
\hline Beryllium (Be) & 4 & 1 & 0.227 & 0.359 & 0.8079 & 0.3435 & 1562 & 3.32 & & 1.149 & 1.04 & \\
\hline Magnesium (Mg) & 12 & 2 & 0.321 & 0.521 & 0.9009 & 0.3211 & 922 & 1.51 & 1.30 & 0.645 & 0.78 & $0.80^{f, g}$ \\
\hline Scandium $(\mathrm{Sc})$ & 21 & 3 & 0.331 & 0.521 & 0.9096 & 0.3257 & 1814 & 3.9 & & 1.341 & 1.87 & $0.96^{c}$ \\
\hline Titanium (Ti) & 22 & 3 & 0.295 & 0.468 & 0.8769 & 0.3264 & 1946 & 4.85 & 3.14 & 1.601 & 2.06 & \\
\hline Cobalt (Co) & 27 & 3 & 0.251 & 0.407 & 0.8338 & 0.3368 & 1770 & 4.39 & & 1.396 & $1.67,1.93$ & \\
\hline Zinc $(\mathrm{Zn})$ & 30 & 2 & 0.266 & 0.495 & 0.8496 & 0.3326 & 692.7 & 1.35 & 1 & 0.511 & 0.47 & $0.54^{c, g}$ \\
\hline Yttrium (Y) & 39 & 3 & 0.365 & 0.573 & 0.9409 & 0.3193 & 1801 & 4.37 & & 1.406 & 1.87 & \\
\hline Zirconium (Zr) & 40 & 4 & 0.323 & 0.515 & 0.9026 & 0.3264 & 2128 & 6.25 & 3.17 & 1.797 & 2.03 & \\
\hline Technetium (Tc) & 43 & 2 & 0.274 & 0.44 & 0.8579 & 0.3305 & 2477 & 6.85 & & 2.060 & 0.25 & \\
\hline Ruthenium (Ru) & 44 & 3 & 0.271 & 0.428 & 0.8554 & 0.3311 & 2527 & 6.74 & & 2.064 & 2.68 & \\
\hline Cadmium (Cd) & 48 & 2 & 0.298 & 0.562 & 0.8799 & 0.3326 & 594.3 & 1.16 & 0.86 & 0.439 & 0.25 & $0.46^{d}$ \\
\hline Gadolinium (Gd) & 64 & 3 & 0.363 & 0.578 & 0.9390 & 0.3122 & 1587 & 4.14 & & 1.282 & & \\
\hline Terbium $(\mathrm{Tb})$ & 65 & 3 & 0.36 & 0.57 & 0.9360 & 0.3130 & 1632 & 4.05 & & 1.288 & & \\
\hline Dysprosium (Dy) & 66 & 3 & 0.359 & 0.565 & 0.9352 & 0.3137 & 1684 & 3.04 & & 1.158 & & \\
\hline Holmium (Ho) & 67 & 3 & 0.358 & 0.562 & 0.9343 & 0.3139 & 1745 & 3.14 & & 1.198 & & \\
\hline Erbium (Er) & 68 & 3 & 0.356 & 0.559 & 0.9324 & 0.3138 & 1797 & 3.29 & & 1.242 & & \\
\hline Thulium (Tm) & 69 & 3 & 0.354 & 0.556 & 0.9306 & 0.3147 & 1820 & 2.42 & & 1.121 & & \\
\hline Lutetium (Lu) & 71 & 3 & 0.35 & 0.555 & 0.9270 & 0.3155 & 1938 & 4.43 & & 1.472 & & \\
\hline Hafnium (Hf) & 72 & 4 & 0.319 & 0.505 & 0.8991 & 0.3214 & 2504 & 6.44 & 3.35 & 1.954 & 2.26 & $2.45^{e}$ \\
\hline Rhenium (Re) & 75 & 4 & 0.276 & 0.446 & 0.8597 & 0.3302 & 3459 & 8.03 & & 2.646 & 3.42 & \\
\hline Osmium (Os) & 76 & 3 & 0.274 & 0.432 & 0.8579 & 0.3306 & 3306 & 8.17 & & 2.603 & 3.03 & \\
\hline Thallium (Tl) & 81 & 3 & 0.346 & 0.552 & 0.9234 & 0.3103 & 577 & 1.88 & 1.04 & 0.539 & 0.40 & $0.46^{d, f}$ \\
\hline
\end{tabular}

${ }^{a}$ Ref. [7]; ${ }^{b}$ Ref. [8]; ${ }^{c}$ Ref. [9]; ${ }^{d}$ Ref. [10]; ${ }^{e}$ Ref. [11]; ${ }^{f}$ Ref. [12]; ${ }^{g}$ Ref. [13]

Variation of the Ashcroft parameter with ECFs for different hcp crystals.

TABLE II

\begin{tabular}{|c|c|c|c|c|c|c|c|c|c|c|}
\hline \multirow{2}{*}{$\begin{array}{l}\text { hcp } \\
\text { crystal }\end{array}$} & \multicolumn{10}{|c|}{$r_{c}$ in AU fitted to $\left(E_{\mathrm{F}}^{1 v}\right)_{\exp }$ or $\left(E_{\mathrm{F}}^{1 v}\right)_{\mathrm{fit}}$} \\
\hline & K-K & Sham & G-V & Kle & Harr & $\mathrm{V}-\mathrm{S}$ & Tay & Hub & M-D & Mean \\
\hline Beryllium (Be) & 1.3466 & 1.3473 & 1.3538 & 1.3427 & 1.3930 & 1.3585 & 1.3408 & 1.4384 & 1.4154 & 1.3707 \\
\hline Magnesium (Mg) & 1.3590 & 1.3551 & 1.3648 & 1.3792 & 1.3912 & 1.3975 & 1.4208 & 1.4188 & 1.4751 & 1.3957 \\
\hline Scandium (Sc) & 1.3849 & 1.3853 & 1.3916 & 1.4136 & 1.4210 & 1.4320 & 1.4501 & 1.4505 & 1.5054 & 1.4260 \\
\hline Titanium (Ti) & 1.2429 & 1.2506 & 1.2512 & 1.2727 & 1.2758 & 1.2790 & 1.2954 & 1.3005 & 1.3420 & 1.2789 \\
\hline Cobalt (Co) & 0.8856 & 0.9025 & 0.8979 & 0.9534 & 0.9244 & 0.9595 & 0.9916 & 0.9447 & 1.0618 & 0.9468 \\
\hline Zinc (Zn) & 0.9432 & 0.9447 & 0.9512 & 0.9946 & 0.9737 & 1.0067 & 1.0487 & 0.9951 & 1.1168 & 0.9972 \\
\hline Yttrium (Y) & 1.6359 & 1.6328 & 1.6409 & 1.6528 & 1.6633 & 1.6694 & 1.6822 & 1.6864 & 1.7224 & 1.6651 \\
\hline Zirconium (Zr) & 1.4970 & 1.5017 & 1.5022 & 1.5468 & 1.5278 & 1.5483 & 1.5902 & 1.5530 & 1.6622 & 1.5477 \\
\hline Tech & 1.3090 & 1.3103 & 1.3122 & 1.3168 & 1.3305 & 1.3199 & 1.3254 & 1.3507 & 1.3529 & 1.3253 \\
\hline Ruthenium (Ru) & 1.1420 & 1.1517 & 1.1485 & 1.1706 & 1.1722 & 1.1717 & 1.1853 & 1.1954 & 1.2279 & 1.1739 \\
\hline Cadmiu & 1.1015 & 1.0985 & 1.1069 & 1.1488 & 1.1290 & 1.1757 & 1.2200 & 1.1506 & 1.2960 & 1.1586 \\
\hline Gadolini & 1.6128 & 1.6103 & 1.6184 & 1.6311 & 1.6389 & 1.6506 & 1.6686 & 1.6655 & 1.7129 & 1.6455 \\
\hline Terbium (Tb) & 1.5955 & 1.5930 & 1.6015 & 1.6150 & 1.6241 & 1.6313 & 1.6497 & 1.6485 & 1.6930 & 1.6279 \\
\hline Dysprosium (D & 1.5714 & 1.5685 & 1.5769 & 1.5920 & 1.6027 & 1.6101 & 1.6269 & 1.6272 & 1.6737 & 1.6055 \\
\hline Holmium (Ho) & 1.5697 & 1.5675 & 1.5750 & 1.5899 & 1.6007 & 1.6070 & 1.6228 & 1.6250 & 1.6684 & 1.6029 \\
\hline Erbium (Er) & 1.5637 & 1.5614 & 1.5689 & 1.5829 & 1.5937 & 1.6001 & 1.6156 & 1.6179 & 1.6605 & 1.5961 \\
\hline Thu & 1.5317 & 1.5300 & 1.5383 & 1.5587 & 1.5684 & 1.5761 & 1.5934 & 1.5944 & 1.6415 & 1.5703 \\
\hline Lutetium (Lu) & 1.5589 & 1.5573 & 1.5635 & 1.5774 & 1.5862 & 1.5916 & 1.6077 & 1.6088 & 1.6491 & 1.5889 \\
\hline Hafnium (Hf) & 1.4356 & 1.4381 & 1.4393 & 1.4801 & 1.4583 & 1.4874 & 1.5242 & 1.4733 & 1.5715 & 1.4786 \\
\hline Rhen & 1.2260 & 1.2368 & 1.2306 & 1.2757 & 1.2587 & 1.2691 & 1.3046 & 1.2830 & 1.3508 & 1.2706 \\
\hline Osmium (Os) & 1.2088 & 1.2135 & 1.2118 & 1.2273 & 1.2300 & 1.2275 & 1.2353 & 1.2516 & 1.2736 & 1.2310 \\
\hline Thallium (Tl) & 1.3945 & 1.3925 & 1.4033 & 1.4316 & 1.4409 & 1.4574 & 1.4862 & 1.4749 & 1.5578 & 1.4488 \\
\hline
\end{tabular}


of $\left(E_{\mathrm{F}}^{1 v}\right)_{T h}$ for cobalt by Angsten et al. [8]. However for zinc the experimental value of $\left(E_{\mathrm{F}}^{1 v}\right)_{\exp }$ is higher than all the theoretical estimates and average value of $r_{c}$ is very close to one AU.

I started computing defect energy calculation using simple model of Ashcroft which contains only one parameter. The complete set of value of $r_{c}$ for cubic crystals will be used for further calculation of energetic of self and impurity diffusion via vacancy mechanism or other type of point defects. The work with two-parameter HeineAbarenkov model is under progress.

\section{References}

[1] A. Ghorai, Def. Diff. Forum 329, 81 (2012).

[2] A. Ghorai, Phys. Rev. B 46, 5229 (1992).

[3] W.A. Harrison, Pseudopotentials in the Theory of Metals, Benjamin, New York 1966.
[4] Q.S. Mei, K. Lu, Philos. Mag. Lett. 88, 203 (2008).

[5] Lianwen Wang, Metals 2014, 570 (2014).

[6] N.W. Ashcroft, Phys. Lett. 23, 48 (1966).

[7] C. Kittel, Introduction to Solid State Physics, 5th ed., Wiley Eastern, New Delhi 1979.

[8] T. Angsten, T. Mayeshiba, H. Wu, D. Morgan, New J. Phys. 16, 015018 (2014).

[9] S. Chattopadhyay, A. Ghorai, in: Proc. CMDays 2014, held at Dept. of Phys., University of Calcutta, 2014.

[10] D. Bhattacharya, A. Ghorai, in: Proc. CMDays 2014, held at Dept. of Phys., University of Calcutta, 2014.

[11] G. Vérité, F. Willaime, Chu Chun Fu, Solid State Phenom. 129, 75 (2007).

[12] P. Tzanetakis, J. Hillairet, G. Revel, Phys. Status Solidi B 75, 433 (1976).

[13] E.V. Chulkov, Sov. Phys. J. 25, 191 (1982). 